\title{
Enhancing damping of gas bearings using linear parameter-varying control
}

\author{
Theisen, Lukas Roy Svane; Niemann, Hans Henrik; Galeazzi, Roberto; Santos, IImar
}

Published in:

Journal of Sound and Vibration

Link to article, DOI:

10.1016/j.jsv.2017.02.021

Publication date:

2017

Document Version

Peer reviewed version

Link back to DTU Orbit

Citation (APA):

Theisen, L. R. S., Niemann, H. H., Galeazzi, R., \& Santos, I. (2017). Enhancing damping of gas bearings using linear parameter-varying control. Journal of Sound and Vibration, 365, 48-64.

https://doi.org/10.1016/j.jsv.2017.02.021

\section{General rights}

Copyright and moral rights for the publications made accessible in the public portal are retained by the authors and/or other copyright owners and it is a condition of accessing publications that users recognise and abide by the legal requirements associated with these rights.

- Users may download and print one copy of any publication from the public portal for the purpose of private study or research.

- You may not further distribute the material or use it for any profit-making activity or commercial gain

- You may freely distribute the URL identifying the publication in the public portal

If you believe that this document breaches copyright please contact us providing details, and we will remove access to the work immediately and investigate your claim 


\title{
Enhancing damping of gas bearings using linear parameter-varying control
}

\author{
Lukas R.S. Theisen ${ }^{\mathrm{a}, *}$, Hans H. Niemann ${ }^{\mathrm{a}}$, Roberto Galeazzi ${ }^{\mathrm{a}}$, Ilmar F. Santos ${ }^{\mathrm{b}}$ \\ ${ }^{a}$ Department of Electrical Engineering, Technical University of Denmark, DK 2800 Kgs. Lyngby, Denmark \\ ${ }^{\mathrm{b}}$ Department of Mechanical Engineering, Technical University of Denmark, DK 2800 Kgs. Lyngby, Denmark
}

\section{A R T I C L E I N F O}

\section{Article history:}

Received 6 May 2016

Received in revised form

16 December 2016

Accepted 7 February 2017

Handling Editor: A.V. Metrikine

\section{Keywords:}

Controllable gas bearings

Experimental validation

Linear parameter-varying control

Rotordynamics

\begin{abstract}
A B S T R A C T
Journal bearings can be lubricated through controllable injectors using pressurised fluids, whose viscosity highly determines the dynamic responses of the rotating machine. The use of fluids with low viscosity is attracting a growing interest due to the reduced friction forces and consequent losses when the machine is in operation. However low viscosity also entails poor damping properties, which may lead to degraded performance or even instability when the rotating machine operates at or near one of the modal frequencies. This issue can be properly addressed by employing active feedback control systems to regulate the injection pressure of the fluid. Due to the strong dependencies of system performance on system parameters, the sought controller should be robust over a large range of operational conditions. This paper addresses the damping enhancement of controllable gas bearings through robust control approaches. Through an extensive experimental campaign the paper evaluates two robust controllers, a linear parametervarying (LPV) controller and $\mathcal{H}_{\infty}$ controller, on their capability to guarantee stability and performance of a gas bearing across the large operational envelopes in rotational speed and injection pressure. The control systems are designed applying state-of-the-art methods in the respective areas. The experimental results clearly demonstrate the feasibility of enhancing the damping properties of a gas bearing by means of robust control methods.
\end{abstract}

(c) 2017 Elsevier Ltd All rights reserved.

\section{Introduction}

High-speed rotating machines are at the core of many industrial processes where energy losses reduction and increased performance are in high demand. One key machine element influencing both losses and performance is the bearing due to its friction characteristics determined by the employed technology [1]. Energy losses due to friction can be reduced by utilizing lubricants with low viscosity; however this worsens the damping properties of the bearing that may determine degraded performance. Pressurised air indeed has low viscosity and, due to its low environmental footprint, increasing research activities are focusing on the design of gas bearings with controllable injection [2-4].

Air bearings have been shown to have poor damping properties [5] that cause significant amplification of the induced vibrations from mass imbalance and external disturbances near the system resonance frequencies. To enable the reliable use

\footnotetext{
* Corresponding author.

E-mail addresses: lrst@elektro.dtu.dk (L.R.S. Theisen), hhn@elektro.dtu.dk (H.H. Niemann), rg@elektro.dtu.dk (R. Galeazzi), ifs@mek.dtu.dk (I.F. Santos).
} 
of this clean technology in rotating machines operating across a wide range of angular velocities there is the need of passive or active systems capable of enhancing the damping to reduce the sensitivity towards disturbances.

\subsection{Literature survey}

Mechatronic systems are an attractive active solution where electromechanical actuators are embedded into the bearings with advanced control systems. A promising design solution is the controllable gas bearing where pressurised air is injected through piezo-actuators [4,6,7]. Active gas bearings are attractive for a number of applications including compressors, atomisers and turbochargers, but the bearing development is still in its early stage. The low damping characteristics are among the factors that prevent employing controllable gas bearings in industrial applications; hence the research has focused on methods for enhancing the damping using feedback control.

The first controllable air bearing design [8] demonstrated that the bearing stiffness varied with the position of a pad. A design solution with active inherent restrictors [3] showed the feasibility of reducing vibrations with a proportional-integral (PI) controller. Another design featuring the radial injection of pressurised air through piezo-actuated injectors [6] illustrated the possibility of increasing the damping by means of a proportional-derivative (PD) controller. The PI and PD gas bearing control systems were tuned for constant angular velocity and pressure of the injected air. However, in industrial applications the bearings are required to operate across a wide range of operating conditions both in terms of rotational speeds of the rotor and air pressure. Therefore control systems designed around a specific operating point may lack robustness to retain performance, and eventually stability, over the working envelope of the machine.

To achieve robustness of the control system the availability of dynamical models and uncertainty descriptions are of paramount importance. Due to the multi-physics nature of the system high-fidelity models as those presented in $[4,9]$ result in large dimensionality (model order higher than hundred), which is generally unsuitable for control system design. Therefore an alternative approach has been explored in [7], where a linear parameter-varying (LPV) identification method was applied to identify low-complexity models of the entire rotor-bearing actuator-sensor system. The obtained model captured the dynamics of the machine and its parameters are scheduled with the shaft angular velocity and air injection pressure. A proportional (P) regulator designed based on the identified model [10] significantly improved the damping at a given operating condition. However, robustness was still not in focus and the controller's performance deteriorated as the operating conditions changed.

LPV control system design represents a suitable methodology when robust stability and performance in the $\mathcal{H}_{\infty}$ sense is sought [11-13]. LPV control has already found a wide application in rotor dynamics for different types of bearing technologies, such as active magnetic bearings (AMBs) [14-16] and oil bearings supported by AMB [14]. In [15] an LPV controller was designed for an AMB to reject the synchronous mass imbalance response. The shaft was assumed rigid to neglect the flexible modes. The work was extended in [16] by using a flexible shaft model and a switching between multiple unstable LPV controllers was proposed. A similar procedure was used in [17]. The LPV controllers were compared in [16,17] to a set of narrow-band $\mathcal{H}_{\infty}$ controllers, which were optimised for mass imbalance rejection at a single angular velocity, and the LPV controller was shown superior in performance. In industrial environments, the machines may further be subject to significant disturbances from mechanical shocks and vibrations from other machinery. Such disturbances may act over a wide frequency range. Critical disturbances are those acting near the underdamped resonance frequencies where strong vibration amplifications occur. The impact of these disturbances can be diminished by proper shaping of the closed-loop sensitivity function.

\subsection{Contributions}

The present work addresses the enhancement of the damping properties of controllable gas bearings through robust control approaches in order to ensure stability and performance over a wide range of operational conditions of the rotating machine. State-of-the-art methods in LPV and $H_{\infty}$ control theories are applied to design two robust regulators according to the mixed sensitivity set-up. These control systems represent a clear technological advancement in the field of gas bearings, where so far only P, PI, PD and LQG regulators were designed and tested [3,6,10,9].

Through an extensive experimental campaign on a scale model of a gas bearing the paper demonstrates the feasibility of reducing the disturbance sensitivity of the rotor dynamics over the operational envelop of the rotating machine by means of damping enhancement. The two robust control systems achieve the design objectives with the LPV regulator doing it with less control effort than the $H_{\infty}$ controller.

This experimental evidence provides solid scientific support of how advanced robust control systems can make gas bearing technology meet the demands of reliability and efficiency of ever changing complex industrial environments where dependability of the control system is a must.

\subsection{Structure of the paper}

The paper is structured as follows: Section 2 provides an overview of the experimental test rig and the identificationbased gas bearing model. Section 3 presents the design methodology of the LPV and $\mathcal{H}_{\infty}$ controllers, whose effectiveness is then investigated through simulations in Section 4. Section 5 provides the assessment of the controllers' performance based on experimental closed-loop data collected over the operating range. Section 6 draws some conclusions. 


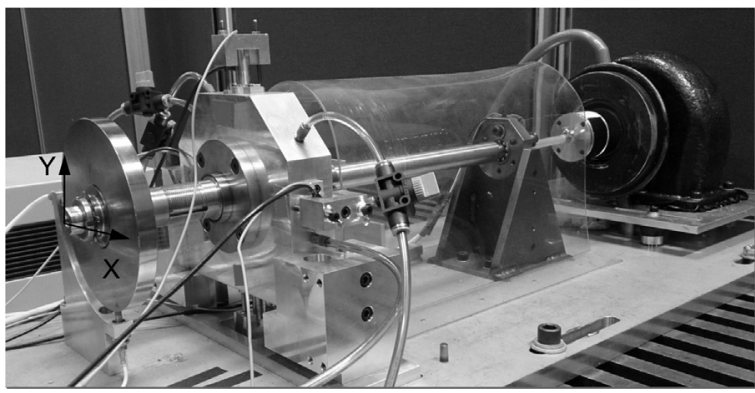

Fig. 1. Experimental test rig: in the gas bearing four piezo-actuated injectors control the injection of pressurised air and the lateral disc movement is measured with eddy-current sensors.

\section{Gas bearing test rig}

This section provides a brief description of the controllable gas bearing test rig. The interested reader is referred to [18] for a detailed analysis of the experimental set-up and the corresponding identified LPV model.

\subsection{Test rig description}

The gas bearing test rig consists of a flexible shaft supported by a ball bearing and the controllable gas bearing, as shown in Fig. 1. A disc is mounted at the end of the shaft to preload it, and the horizontal and vertical disc movements are measured with eddy-current sensors. The deviation from the equilibrium position is denoted by $\mathbf{p}=\left[p_{x}, p_{y}\right]^{\mathrm{T}}$, where the horizontal $(x)$, and the vertical $(y)$ shaft directions are according to the reference frame sketched in the photo. The shaft is actuated by the injection of pressurised air through four controllable piezo-actuated injectors mounted in the gas bearing. The airflow generates a fluid film that provides a stable lifting force on the shaft. This fluid film gives rise to dynamics in the rotorbearing coupling, which is a function of both the shaft angular velocity and the injection pressure [4].

The piezo-actuators are controlled pairwise and have local control loops as described in [7] to linearise their dynamics. Thereby one piezo-actuator reference $r_{x}$ is used to control the position of the horizontal injectors and one reference $r_{y}$ is used to control the vertical ones. These references take values in the interval $[-5,5] \mathrm{V}$, which corresponds to full-span motion of the piezo-actuator positions in the interval $[0,45] \mu \mathrm{m}$. A turbine drives the shaft and an encoder measures the angular position $\phi$, from which the angular velocity $q_{1}$ is calculated. The pressurised air to the gas bearing is supplied from a large pressure tank. In an industrial application, the injection pressure would be slowly time-varying. For the test rig at hand, a lever is mounted to allow manual control of the pressure variations. The injection pressure $q_{2}$ is measured before the airflow is split up to the four actuators.

\subsection{Gas bearing model}

Gas bearing models derived from first principles [4,9] rely on solution schemes to the nonlinear partial differential modified Reynolds equation [19]. Those methods often produce high order models and are subject to parameter uncertainty [9]. These multi-physics models are high-fidelity; however their high dimensionality introduce a large computational burden and make them unsuitable for control system design. To overcome these issues the authors in [7] adopted a grey-box system identification procedure to obtain linear parameter-varying models of the physical test rig. The experimentally identified LPV model captures the dominant dynamics of the rotating machine while retaining a reduced complexity that suits the design of control systems [10]. The method from [7] is summarised in the following.

The gas bearing operational profile is scheduled with respect to the shaft angular velocity $q_{1}$ and the injection pressure $q_{2}$, which define the scheduling parameter vector $\mathbf{q}=\left[q_{1}, q_{2}\right]^{\mathrm{T}}$. The scheduling parameters can vary in the intervals $q_{1} \in\left[\underline{q}_{1}, \bar{q}_{1}\right]=[0,6] \mathrm{krpm}, q_{2} \in\left[\underline{q}_{2}, \bar{q}_{2}\right]=[0.3,0.7] \mathrm{MPa}$. Local LTI models are identified from data-sets collected in correspondence of the grid points $q_{1} \in\{0,1,2,3,4,5,6\} \mathrm{krpm}, q_{2} \in\{0.3,0.4,0.5,0.6,0.7\} \mathrm{MPa}$. The local models are identified with a structure that allows interpolation of the model parameters to obtain the LPV model. The control input vector contains the position references sent to the piezo-actuators $\mathbf{r}=\left[r_{x}, r_{y}\right]^{\mathrm{T}}$ and the output vector to be controlled is the lateral disc movement $\mathbf{p}=\left[p_{x}, p_{y}\right]^{\mathrm{T}}$. The control input was selected as a pseudo-random step-wise excitation.

The model is a cascade interconnection of two subsystems: the LTI actuator dynamics and the parameter-varying rotorbearing dynamics. The actuator dynamics $\mathbf{G}_{a c t}$ is: 
Table 1

Actuator model parameters.

\begin{tabular}{lllllll}
\hline $\begin{array}{l}\text { Parameter } \\
\text { Units }\end{array}$ & $\begin{array}{l}p_{1, x} \\
\mathrm{krad} / \mathrm{s}\end{array}$ & $\begin{array}{l}p_{2, x} \\
\mathrm{krad} / \mathrm{s}\end{array}$ & $\begin{array}{l}\kappa_{x} \\
-\end{array}$ & $\begin{array}{l}p_{1, y} \\
\mathrm{krad} / \mathrm{s}\end{array}$ & $\begin{array}{l}p_{2, y} \\
\mathrm{krad} / \mathrm{s}\end{array}$ \\
\hline Value & 3.08 & 8.14 & 1.86 & 2.45 & 6.49 \\
\hline
\end{tabular}

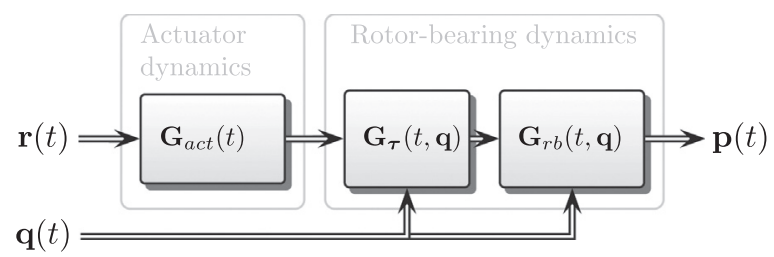

Fig. 2. Overview of the gas bearing model, which includes the actuator dynamics and parameter-varying rotor-bearing dynamics.

$$
\mathbf{G}_{a c t}(s)=\left[\begin{array}{cc}
G_{a, x}(s) & 0 \\
0 & G_{a, y}(s)
\end{array}\right], \quad G_{a, j}(s)=\frac{\kappa_{a, j}}{\left(\frac{1}{p_{1, j}} s+1\right)\left(\frac{1}{p_{2, j}} s+1\right)}, \quad j \in\{x, y\}
$$

The actuator model parameters are listed in Table 1. The rotor-bearing dynamics is modelled as the interconnection of parameter-varying Padé approximated input delays and a second order parameter-varying mass-spring-damper system with stiffness, damping and input gains $\tilde{\mathcal{K}}, \tilde{\mathcal{D}}, \tilde{\mathcal{B}}$ and a unit mass $\tilde{\mathcal{M}}$. The equivalent stiffness, damping and input gain are then defined as $\mathcal{K} \triangleq \tilde{\mathcal{M}}^{-1} \tilde{\mathcal{K}}, \mathcal{D} \triangleq \tilde{\mathcal{M}}^{-1} \tilde{\mathcal{D}}$ and $\mathcal{B} \triangleq \tilde{\mathcal{M}}^{-1} \tilde{\mathcal{B}}$, respectively.

Let the disc position $\mathbf{p}(t)$ and velocity $\dot{\mathbf{p}}(t)$ be the state variables, then the state-space realisation reads

$$
\mathbf{G}_{r b}(t, \mathbf{q})=\left\{\begin{array}{l}
{\left[\begin{array}{l}
\dot{\mathbf{p}}(t) \\
\ddot{\mathbf{p}}(t)
\end{array}\right]=\left[\begin{array}{cc}
\mathbf{0} & \mathbf{I} \\
\mathcal{K}(\mathbf{q}) & \mathcal{D}(\mathbf{q})
\end{array}\right]\left[\begin{array}{c}
\mathbf{p}(t) \\
\dot{\mathbf{p}}(t)
\end{array}\right]+\left[\begin{array}{c}
\mathbf{0} \\
\mathcal{B}(\mathbf{q})
\end{array}\right] \mathbf{u}_{r b}(t)} \\
\mathbf{y}(\mathbf{t})=\left[\begin{array}{ll}
\mathbf{I} & \mathbf{0}
\end{array}\right]\left[\begin{array}{c}
\mathbf{p}(t) \\
\dot{\mathbf{p}}(t)
\end{array}\right]
\end{array}\right.
$$

The parameter-varying matrices are quadratic polynomials of the scheduling parameter vector q:

$$
\begin{aligned}
& \mathcal{K}(\mathbf{q})=\mathcal{K}_{0}+\mathcal{K}_{1} q_{1}+\mathcal{K}_{2} q_{2}+\mathcal{K}_{3} q_{1}^{2}+\mathcal{K}_{4} q_{2}^{2}+\mathcal{K}_{5} q_{1} q_{2} \\
& \mathcal{D}(\mathbf{q})=\mathcal{D}_{0}+\mathcal{D}_{1} q_{1}+\mathcal{D}_{2} q_{2}+\mathcal{D}_{3} q_{1}^{2}+\mathcal{D}_{4} q_{2}^{2}+\mathcal{D}_{5} q_{1} q_{2} \\
& \mathcal{B}(\mathbf{q})=\mathcal{B}_{0}+\mathcal{B}_{1} q_{1}+\mathcal{B}_{2} q_{2}+\mathcal{B}_{3} q_{1}^{2}+\mathcal{B}_{4} q_{2}^{2}+\mathcal{B}_{5} q_{1} q_{2}
\end{aligned}
$$

where $\mathcal{K}_{0}, \ldots, \mathcal{K}_{5}, \ldots, \mathcal{B}_{5}$ describe the development within the operating range. The two Padé approximated input delays $-\tau_{x}$ in the horizontal actuation and $\tau_{y}$ in the vertical one - are included in the identification and are also parameter-dependent:

$$
\begin{aligned}
& \tau_{x}(\mathbf{q})=\tau_{x, 0}+\tau_{x, 1} q_{1}+\tau_{x, 2} q_{2}+\tau_{x, 3} q_{1}^{2}+\tau_{x, 4} q_{2}^{2}+\tau_{x, 5} q_{1} q_{2} \\
& \tau_{y}(\mathbf{q})=\tau_{y, 0}+\tau_{y, 1} q_{1}+\tau_{y, 2} q_{2}+\tau_{y, 3} q_{1}^{2}+\tau_{y, 4} q_{2}^{2}+\tau_{y, 5} q_{1} q_{2}
\end{aligned}
$$

The delay approximations have state-space realisations:

$\mathbf{G}_{\boldsymbol{\tau}}(t, \mathbf{q})=\left[\begin{array}{c|c}-2 \boldsymbol{\tau}(\mathbf{q})^{-1} & 1 \\ \hline 4 \boldsymbol{\tau}(\mathbf{q})^{-1} & -1\end{array}\right], \quad j \in\{x, y\}$

in which $\boldsymbol{\tau}(\mathbf{q})=\operatorname{diag}\left(\tau_{x}(\mathbf{q}), \tau_{y}(\mathbf{q})\right)$. Fig. 2 provides an overview of the model and the parameter-dependencies.

It is of interest to reduce the model order, since this decreases the controller order for synthesis with robust control techniques. Residualisation [20] is therefore used to reduce the model from 10 th to 6th order. The actuator dynamics is then approximated as constant gain matrix

$$
\mathbf{G}_{a c t}(S) \approx \mathcal{N}, \quad \mathcal{N}=\left[\begin{array}{cc}
\kappa_{a, x} & 0 \\
0 & \kappa_{a, y}
\end{array}\right]
$$

The gas bearing model can then be formulated in state-space as 
Table 2

Derived parameters' variations.

\begin{tabular}{llc}
\hline Parameter & Interval & Deviation $^{\mathrm{a}}(\%)$ \\
\hline Horizontal natural frequency $\omega_{x}$ & {$[657,775] \mathrm{rad} / \mathrm{s}$} & 15.2 \\
Vertical natural frequency $\omega_{y}$ & {$[736,832] \mathrm{rad} / \mathrm{s}$} & 11.5 \\
Horizontal damping factor $\zeta_{x}$ & {$[0.033,0.12]$} & 73.2 \\
Vertical damping factor $\zeta_{y}$ & {$[0.016,0.092]$} & 82.7 \\
Horizontal delay $\tau_{x}$ & {$[0.056,0.41] \mathrm{ms}$} & 86.5 \\
Vertical delay $\tau_{x}$ & {$[0.093,0.46] \mathrm{ms}$} & 80.0 \\
Static gain $g_{x x}$ & {$[2.37,3.27] \mu \mathrm{m} / \mathrm{V}$} & 27.4 \\
Static gain $g_{x y}$ & {$[-0.01,0.74] \mu \mathrm{m} / \mathrm{V}$} & 101.5 \\
Static gain $g_{y x}$ & {$[-0.61,0.14] \mu \mathrm{m} / \mathrm{V}$} & 540.8 \\
Static gain $g_{y y}$ & {$[2.97,3.24] \mu \mathrm{m} / \mathrm{V}$} & 8.2 \\
\hline
\end{tabular}

${ }^{a}$ Deviation from the maximum value for variable $a \in[\underline{a}, \bar{a}]$ calculated as $100(\bar{a}-\underline{a}) / \bar{a}$.
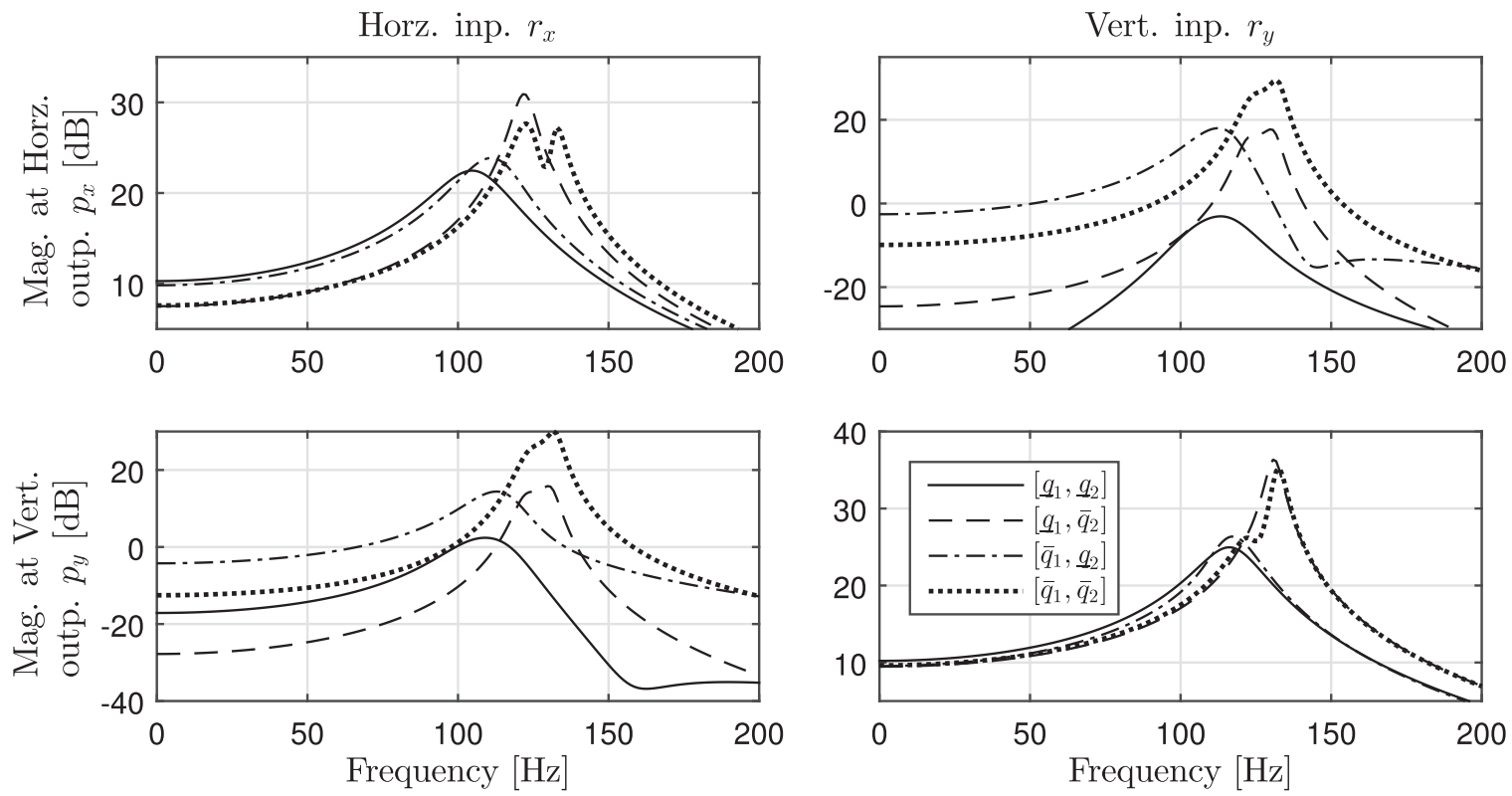

Fig. 3. Bode diagrams of the gas bearing system for the scheduling parameter extrema $\left[\underline{q}_{1}, \underline{q}_{2}\right],\left[\underline{q}_{1}, \bar{q}_{2}\right],\left[\bar{q}_{1}, \underline{q}_{2}\right],\left[\bar{q}_{1}, \bar{q}_{2}\right]$.

$\mathbf{G}(t)=\left[\begin{array}{ccc|c}0 & \mathbf{I} & \mathbf{0} & \mathbf{0} \\ \mathcal{K}(\mathbf{q}) & \mathcal{D}(\mathbf{q}) & 2 \mathcal{B}(\mathbf{q}) \boldsymbol{\tau}(\mathbf{q})^{-1} & -\mathcal{B}(\mathbf{q}) \mathcal{N} \\ \mathbf{0} & \mathbf{0} & -\boldsymbol{\tau}(\mathbf{q})^{-1} & \mathcal{N} \\ \hline \mathbf{I} & \mathbf{0} & \mathbf{0} & \mathbf{0}\end{array}\right]$

Since the parameters' variation is slow, it is reasonable to assume $\dot{\mathbf{q}} \approx \mathbf{0}$.

In [7] the LPV model was shown to preserve important system properties (natural frequencies, damping factors and static gains) within the desired range of the scheduling parameters. The shape of the time-varying parameters are shown in [7] as functions of the scheduling parameters. Here, the effects of parameters' variations are quantified by a set of derived parameters for the system. These parameters are chosen to describe the static gain matrix $\left.\mathbf{G}(s, \mathbf{q})\right|_{s=0}=\left[\begin{array}{ll}g_{x y} & g_{x y} \\ g_{y x} & g_{y y}\end{array}\right]$ and the eigenvalues. The model has six eigenvalues, two related to the time delays and two complex pole pairs, which model the two first critical speeds of the rotor-bearing. The natural frequencies and damping ratios associated with the complex pole pairs are denoted $\left(\omega_{x}, \omega_{y}\right)$ and $\left(\zeta_{x}, \zeta_{y}\right)$, respectively. Table 2 provides the range of variation of the considered parameters: they all vary significantly across the operating range of the gas bearing.

Fig. 3 shows the variations of the frequency responses of the gas bearing system when the scheduling parameters are at the extrema of the operating range $\left(\left[\underline{q}_{1}, \underline{q}_{2}\right],\left[\underline{q}_{1}, \bar{q}_{2}\right],\left[\bar{q}_{1}, \underline{q}_{2}\right],\left[\bar{q}_{1}, \bar{q}_{2}\right]\right)$. For high angular velocities, the cross coupling gains increase significantly. For high injection pressures, the natural frequencies augment and the damping decreases. 


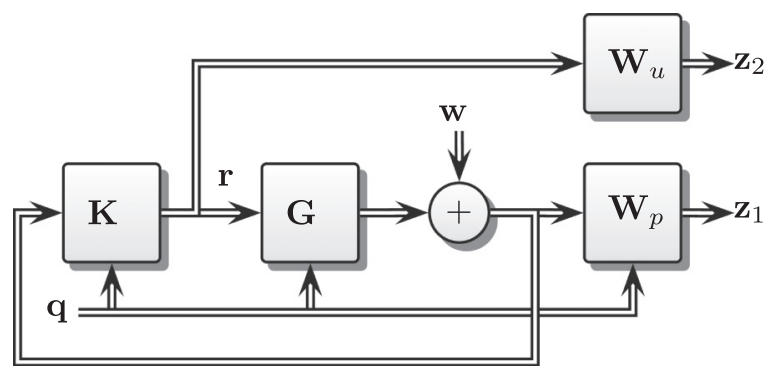

Fig. 4. The augmented plant with controller for LPV controller design with performance weights $\mathbf{W}_{p}$ and controller sensitivity weight $\mathbf{W}_{u}$.

\section{Damping enhancing control of gas bearings}

This section describes the design of the controllers. First a discussion is given of what is meant by damping enhancing control, followed by the design of the LPV controller and the $\mathcal{H}_{\infty}$ controller.

The gas bearing dynamics contains under-damped eigenfrequencies making it very sensitive to disturbances near the associated resonance frequencies. The impact of these disturbances can be diminished by proper shaping of the closed-loop sensitivity function. Consider the general control system from [20], the output $\mathbf{y}(s)$ is given by

$$
\mathbf{y}(s)=\mathbf{G}(s) \mathbf{u}(s)+\mathbf{G}_{d}(s) \mathbf{d}(s)
$$

where $\mathbf{G}(s)$ is the plant to be controlled by means of the input $\mathbf{u}(s)$. The disturbances $\mathbf{d}(s)$ enter the output through the (generally unknown) dynamics $\mathbf{G}_{d}(s)$. When the output feedback control law $\mathbf{u}(s)=-\mathbf{K}(s) \mathbf{y}(s)$ is applied then the system output becomes

$$
\mathbf{y}(s)=\mathbf{S}(s) \mathbf{G}_{d}(s) \mathbf{d}(s), \quad \mathbf{S}(s)=(\mathbf{I}+\mathbf{G}(s) \mathbf{K}(s))^{-1}
$$

The analysis shows that the effects of the disturbances on the output in a specific frequency range $\left(\omega_{\min }, \omega_{\max }\right)$ are diminished by a controller $\mathbf{K}$ that obtains a low sensitivity $|\mathbf{S}(j \omega)| \ll 1$ in that range. Therefore the damping of the resonance frequencies is increased by reducing the sensitivity near the associated frequency.

\subsection{LPV controller design}

The LPV controller $\mathbf{K}$ should enhance the damping to reduce the disturbance sensitivity near the resonance frequencies and at the same time avoid wear of the actuator. These disturbance and noise rejection requirements can be cast according to the mixed sensitivity set-up [20] that seeks to minimise

$$
\mathbf{K}(s, \mathbf{q})=\underset{\gamma}{\operatorname{argmin}}\|\mathbf{N}(s, \mathbf{q})\|_{\infty}, \quad \mathbf{N}(s, \mathbf{q})=\left[\begin{array}{c}
\mathbf{W}_{p}(s) \mathbf{S}(s) \\
\mathbf{W}_{u}(s) \mathbf{K}(s) \mathbf{S}(s)
\end{array}\right],
$$

where $\mathbf{W}_{p}(s)$ and $\mathbf{W}_{u}(s)$ are frequency dependent weighting matrices and the closed-loop sensitivity function is defined for constant $\mathbf{q}=\mathbf{q}_{0}$, i.e. $\mathbf{S}\left(s, \mathbf{q}_{0}\right) \triangleq\left(\mathbf{I}+\mathbf{G}\left(s, \mathbf{q}_{0}\right) \mathbf{K}\left(s, \mathbf{q}_{0}\right)\right)^{-1}$. The external output disturbance $\mathbf{w}$ and the external outputs $\mathbf{z}=\left[\mathbf{z}_{1}^{\mathrm{T}}, \mathbf{z}_{2}^{\mathrm{T}}\right]^{\mathrm{T}}$ are included into the system description to obtain the augmented plant as shown in Fig. 4.

To enhance the damping characteristics, the controller should have high performance in the frequency range around the under-damped eigenfrequencies of the rotor-bearing $\omega_{x}(\mathbf{q})$ and $\omega_{y}(\mathbf{q})$. The performance weighting matrix $\mathbf{W}_{p}(s)$ is therefore designed as

$$
\left.\mathbf{W}_{p}(s)=\operatorname{diag}\left(w_{p h}(s, \mathbf{q}), w_{p v}(s, \mathbf{q})\right)\right)
$$

where $w_{p h}(\mathbf{s}, \mathbf{q})$ and $w_{p v}(\mathbf{s}, \mathbf{q})$ are inverse notch-like filters

$$
\begin{aligned}
& w_{p h}(s, \mathbf{q})=\frac{s^{2}+2 \zeta_{1} \omega_{h}(\mathbf{q}) s+\omega_{h}(\mathbf{q})^{2} k_{0}}{s^{2}+2 \zeta_{2} \omega_{h}(\mathbf{q}) s+\omega_{h}(\mathbf{q})^{2}} \\
& w_{p v}(s, \mathbf{q})=\frac{s^{2}+2 \zeta_{1} \omega_{v}(\mathbf{q}) s+\omega_{v}(\mathbf{q})^{2} k_{0}}{s^{2}+2 \zeta_{2} \omega_{v}(\mathbf{q}) s+\omega_{v}(\mathbf{q})^{2}}
\end{aligned}
$$

The natural frequencies $\omega_{h}(\mathbf{q})=\omega_{x}(\mathbf{q}), \omega_{v}(\mathbf{q})=\omega_{y}(\mathbf{q})$ are chosen as the under-damped natural frequencies of the gas bearing to obtain a high weight around these. The weight at the resonance frequencies is a tuning parameter and an increased peak gain at the resonance frequency in general results in more damping. A few iterations showed that $\zeta_{1}=0.3$ and $\zeta_{2}=0.05$ provide a sufficient damping enhancement over the operating range. The peak gain is then $19 \mathrm{~dB}$. The low sensitivity around the natural frequencies 

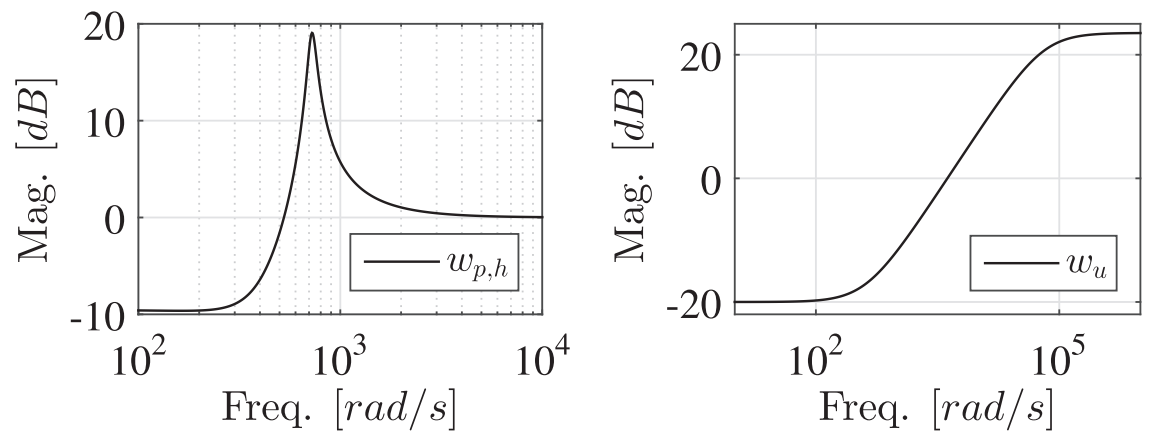

Fig. 5. Example of performance weight $w_{p, h}(s, \mathbf{q})$ as defined in (12) and controller sensitivity weight $w_{u}(s)$ as defined in $(14)$ for scheduling parameter $\mathbf{q}=[2.87 \mathrm{krpm}, 0.51 \mathrm{MPa}]^{\mathrm{T}}$.

comes at the cost of increased sensitivity in another frequency range due to Bode's sensitivity integral [21]. In [10] the authors showed that it is beneficial to place this sensitivity increase in the low frequency range where an amplification of disturbances is acceptable. The constant $k_{0}=1 / 3$ determines the low frequency weight. The control signal sensitivity weighting matrix $\mathbf{W}_{u}(s)$ is chosen to penalise control action at high frequency with the high-pass filter suggested in [20]:

$$
\mathbf{W}_{u}(s)=\mathbf{I} w_{u}(s), \quad w_{u}(s)=\frac{s / M_{b}+\omega_{b}}{s+\omega_{b} A_{b}},
$$

where the low frequency gain is $1 / A_{b}, A_{b}=10$, the high frequency gain is $1 / M_{b}=15$, and the approximate crossover frequency is $\omega_{b}=2 \pi 1000 \mathrm{rad} / \mathrm{s}$. The weight $w_{u}(s)$ and a realisation of the parameter-varying performance weight $w_{p h}(s)$ are shown in Fig. 5 . The augmented plant $\mathbf{P}$ has an LPV state-space realisation of 12 th order (6th order system, 4 th order $\mathbf{W}_{p}$, and 2 nd order $\mathbf{W}_{u}$ )

$\mathbf{P}(\mathbf{q})=\left[\begin{array}{c|cc}\mathbf{A}(\mathbf{q}) & \mathbf{B}_{1} & \mathbf{B}_{2}(\mathbf{q}) \\ \hline \mathbf{C}_{1}(\mathbf{q}) & \mathbf{D}_{11} & \mathbf{D}_{12} \\ \mathbf{C}_{2} & \mathbf{D}_{21} & \mathbf{D}_{22}\end{array}\right]$,

The guidelines proposed in [13] were utilised to determine the synthesis method for the LPV controller. The scheduling parameter dependency is neither affine nor on a linear fractional transformation form. The number of scheduling parameters however is fairly low. For the given system, the guidelines then suggest to use the gridding-based LPV synthesis method [11].

A full-order LPV controller $\mathbf{K}(\mathbf{q})$ for the system $\mathbf{P}(\mathbf{q})$ guarantees $\|\mathbf{z}\|_{2} \leq \gamma\|\mathbf{w}\|_{2}$ if it is a solution of the two matrix inequalities

$$
\mathcal{T}_{1}>\mathbf{0}, \quad \mathcal{T}_{2}<\mathbf{0}
$$

for the decision variables $\hat{\mathbf{A}}_{K}, \hat{\mathbf{B}}_{K}, \hat{\mathbf{C}}_{K}, \mathbf{D}_{K}, \mathbf{X}, \mathbf{Y}, \gamma$ where

$$
\begin{aligned}
\mathcal{T}_{1} & =\left[\begin{array}{cc}
\mathbf{X} & \mathbf{I}_{n_{a}} \\
\mathbf{I}_{n_{a}} & \mathbf{Y}
\end{array}\right], \\
\mathcal{T}_{2} & =\left[\begin{array}{cccc}
\mathbf{X} \mathbf{A}+\hat{\mathbf{B}}_{K} \mathbf{C}_{2}+(\star) & \star & \star & \star \\
\hat{\mathbf{A}}_{K}^{\mathrm{T}}+\mathbf{A}+\mathbf{B}_{2} \mathbf{D}_{K} \mathbf{C}_{2} & \mathbf{A Y}+\mathbf{B}_{2} \hat{\mathbf{C}}_{K}+(\star) & \star & \star \\
\left(\mathbf{X B}_{1}+\hat{\mathbf{B}}_{K} \mathbf{D}_{21}\right)^{\mathrm{T}} & \left(\mathbf{B}_{1}+\mathbf{B}_{2} \mathbf{D}_{K} \mathbf{D}_{21}\right)^{\mathrm{T}} & -\gamma \mathbf{I}_{n_{w}} & \star \\
\mathbf{C}_{1}+\mathbf{D}_{12} \mathbf{D}_{K} \mathbf{C}_{2} & \mathbf{C}_{1} \mathbf{Y}+\mathbf{D}_{12} \hat{\mathbf{C}}_{K} & \mathbf{D}_{11}+\mathbf{D}_{12} \mathbf{D}_{K} \mathbf{D}_{21} & -\gamma \mathbf{I}_{n_{z}}
\end{array}\right]
\end{aligned}
$$

The dependency of the scheduling parameters is omitted in the above.

Problem (16) is a linear matrix inequality (LMI) with infinite dimension, which becomes tractable by applying the following guidelines from [12]: (1) the matrix inequalities are evaluated over a finite grid $Q_{d}$ of scheduling parameters covering the system operating range; (2) the decision variables are constrained to have the same non-linear functional dependency as the plant which is defined by the basis functions $f_{n}(\mathbf{q})$ for $N=5: f_{1}=q_{1}, f_{2}=q_{2}, f_{3}=q_{1}^{2}, f_{4}=q_{2}^{2}, f_{5}=q_{1} q_{2}$. The decision variables are then given by 

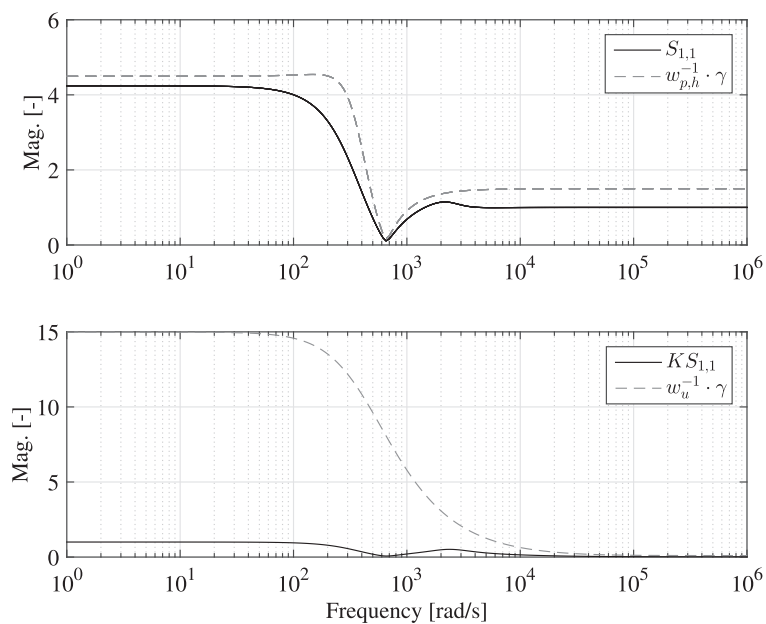

Fig. 6. Sensitivity functions. Upper plot: horizontal output sensitivity $S_{1,1}(s)$ and the corresponding weight $w_{p, h}^{-1}(s) \gamma$. Lower plot: control sensitivity $K S_{1,1}(s)$ and the corresponding weight $w_{u}^{-1}(s) \gamma$.

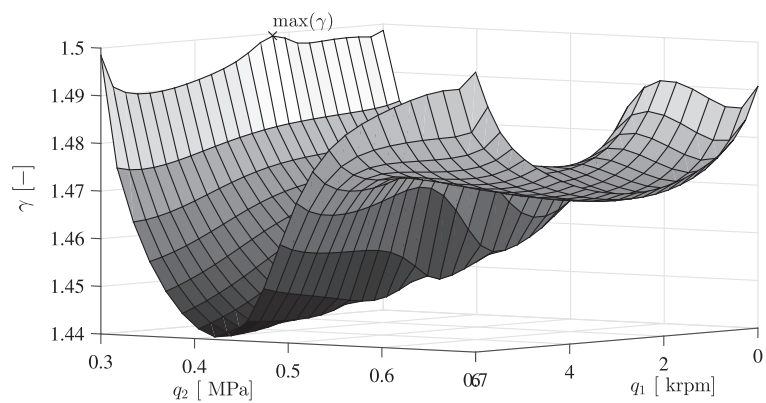

Fig. 7. Validation of LPV controller. Around $q_{2} \approx 0.4 \mathrm{MPa}$ better performance is achieved than defined by $\gamma_{d}$ whereas the limiting performance is obtained at $\mathbf{q}=[2.3 \mathrm{krpm}, 0.3 \mathrm{MPa}]^{\mathrm{T}}$, where $\max (\gamma) / \gamma_{d}=1.00054$.

$$
\begin{array}{ll}
\hat{\mathbf{A}}_{K}(\mathbf{q})=\hat{\mathbf{A}}_{K, 0}+\sum_{n=1}^{N} f_{n}(\mathbf{q}) \hat{\mathbf{A}}_{K, n}, & \hat{\mathbf{B}}_{K}(\mathbf{q})=\hat{\mathbf{B}}_{K, 0}+\sum_{n=1}^{N} f_{n}(\mathbf{q}) \hat{\mathbf{B}}_{K, n} \\
\hat{\mathbf{C}}_{K}(\mathbf{q})=\hat{\mathbf{C}}_{K, 0}+\sum_{n=1}^{N} f_{n}(\mathbf{q}) \hat{\mathbf{C}}_{K, n}, & \mathbf{D}_{K}(\mathbf{q})=\mathbf{D}_{K, 0}+\sum_{n=1}^{N} f_{n}(\mathbf{q}) \mathbf{D}_{K, n}
\end{array}
$$

In (18) $\hat{\mathbf{A}}_{K, j}, \hat{\mathbf{B}}_{K, j}, \hat{\mathbf{C}}_{K, j}$ and $\mathbf{D}_{K, j}, j=0, \ldots, N$, are matrices to be determined by the LMI optimisation.

The augmented system (15) must be in coherent state-space bases in the design grid. To improve its numerical conditioning the plant (15) is balanced using a constant transformation matrix $\mathbf{T}_{0}$, such that $\left[\begin{array}{cc}\mathbf{T}_{0} \mathbf{A} \mathbf{T}_{0}^{-1} & \mathbf{T}_{0} \mathbf{B} \\ \mathbf{C r}_{0}^{-1} & \mathbf{0}\end{array}\right]$ where $\mathbf{T}_{0}$ equalises the maximum row and column norms across the entire array of gridded augmented plants. The similarity transformation is found in Matlab with ssbal.m.

To obtain a controller that is practically valid (i.e. it is not a function of the scheduling parameter derivatives, which are not measured), it is proposed in [12] to constrain either $\mathbf{X}$ or $\mathbf{Y}$ to be constant. All attempts trying to constrain $\mathbf{X}$ to a constant failed to provide a feasible LMI solution. We therefore chose $\mathbf{X}$ to be function of the scheduling parameters and $\mathbf{Y}$ to be constant

$$
\mathbf{X}(\mathbf{q})=\mathbf{X}_{0}+\sum_{n=1}^{N} f_{n}(\mathbf{q}) \mathbf{X}_{n}, \quad \mathbf{Y}=\mathbf{Y}_{0}
$$

The resulting LMIs are then solved to minimise the objective function

$$
\min \left\{\gamma+\varepsilon_{T} \sum_{n=0}^{N} \operatorname{tr}\left(\mathbf{X}_{n}\right)+\varepsilon_{T} \operatorname{tr}\left(\mathbf{Y}_{0}\right)\right\}
$$

where the small constant $\varepsilon_{T}=2.2 \cdot 10^{-16}$ is chosen to avoid numerically large values in $\mathbf{X}$ and $\mathbf{Y}$. 


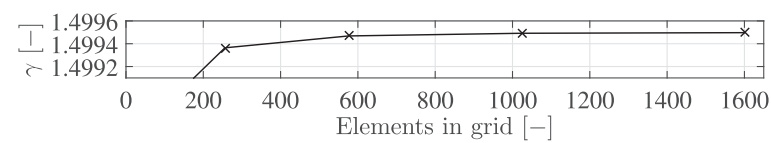

Fig. 8. Performance index for varying validation grid sizes. The performance converges as the grid size increases.

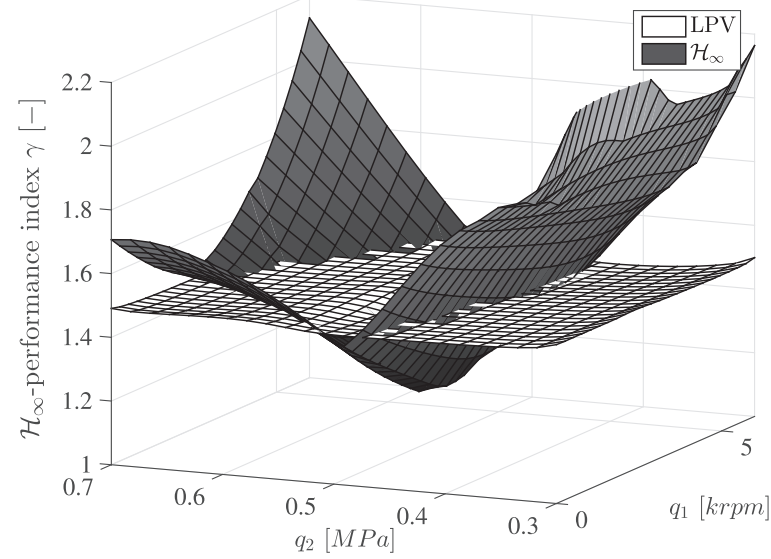

Fig. 9. Comparison of performance index $\gamma$ for the $\mathcal{H}_{\infty}$ and LPV controllers evaluated in the validation grid $Q_{v}$.

The LMIs are generated in Matlab with YALMIP [22], which only permits non-strict LMIs. Therefore the matrix inequalities (16) are relaxed to

$$
\mathcal{T}_{1}>\varepsilon, \quad \mathcal{T}_{2} \prec-\varepsilon,
$$

where $\varepsilon>0$ is some small positive number. Design iterations show that the value $\varepsilon=1.5 \cdot 10^{-3}$ and a grid of $8 \times 8$ equidistant values for the scheduling parameter provide an adequate solution. The LMIs are solved over the design grid $Q_{d}$ using MOSEK to obtain a solution satisfied for $\gamma=\gamma_{d}=1.4987$. Fig. 6 shows a representative example of the closed-loop performance, which confirms that the output sensitivity $\mathbf{S}(s)$ is indeed low in the frequency range nearby the open-loop resonance frequency, and the control sensitivity $\mathbf{K S}(s)$ is low for high frequencies as desired.

To ensure robust performance over the whole scheduling parameter interval, the LMI solution must be validated over a finer validation grid $Q_{v}$. It is common that the value $\gamma_{d}$ must be increased for the LMI solution to be valid in the validation grid. Fig. 7 shows how the performance index $\gamma$ varies when the parameters $q_{1}$ and $q_{2}$ are scheduled across the validation grid of $16 \times 16$ equidistant values. These grid values are listed in Appendix B. This process was repeated for increasing number of grid points to investigate the sensitivity of the performance index $\gamma$ with respect to the grid size. Fig. 8 shows that the performance index converges to the constant value $\gamma=1.4995$ as the validation grid size increases.

From the solution, the factorisation problem $\mathbf{I}-\mathbf{X Y}=\mathbf{N M}^{\mathbf{T}}$ must be solved for $\mathbf{N}(\mathbf{q})$ and $\mathbf{M}(\mathbf{q})$. We choose $\mathbf{M}=\mathbf{I}$ and $\mathbf{N}(\mathbf{q})=\mathbf{I}-\mathbf{X}(\mathbf{q}) \mathbf{Y}_{0}$. The controller parameters are then calculated as

$\mathbf{K}(\mathbf{q})=\left[\begin{array}{l|l}\mathbf{A}_{K}(\mathbf{q}) & \mathbf{B}_{K}(\mathbf{q}) \\ \hline \mathbf{C}_{K}(\mathbf{q}) & \mathbf{D}_{K}(\mathbf{q})\end{array}\right]$

in which:

$$
\begin{aligned}
& \mathbf{A}_{K}=\mathbf{N}^{-1}\left(\mathbf{X} \dot{\mathbf{Y}}+\mathbf{N} \dot{\mathbf{M}}^{\mathrm{T}}+\hat{\mathbf{A}}_{K}-\mathbf{X}\left(\mathbf{A}-\mathbf{B}_{2} \mathbf{D}_{K} \mathbf{C}_{2}\right) \mathbf{Y}-\hat{\mathbf{B}}_{K} \mathbf{C}_{2} \mathbf{Y}-\mathbf{X} \mathbf{B}_{2} \hat{\mathbf{C}}_{K}\right) \mathbf{M}^{-\mathrm{T}} \\
& \mathbf{B}_{K}=\mathbf{N}^{-1}\left(\hat{\mathbf{B}}_{K}-\mathbf{X} \mathbf{B}_{2} \mathbf{D}_{K}\right) \\
& \mathbf{C}_{K}=\left(\hat{\mathbf{C}}_{K}-\mathbf{D}_{K} \mathbf{C}_{2} \mathbf{Y}\right) \mathbf{M}^{-\mathrm{T}}
\end{aligned}
$$

where the matrices are functions of the scheduling parameters. The evaluation of the matrices $\hat{\mathbf{A}}_{K}, \hat{\mathbf{B}}_{K}, \hat{\mathbf{C}}_{K}, \hat{\mathbf{D}}_{K}, \mathbf{X}, \mathbf{Y}$ and the calculation of the controller matrices (23) have to be performed online for each scheduling sampling step, which is computationally heavy due to the two matrix inversions. 

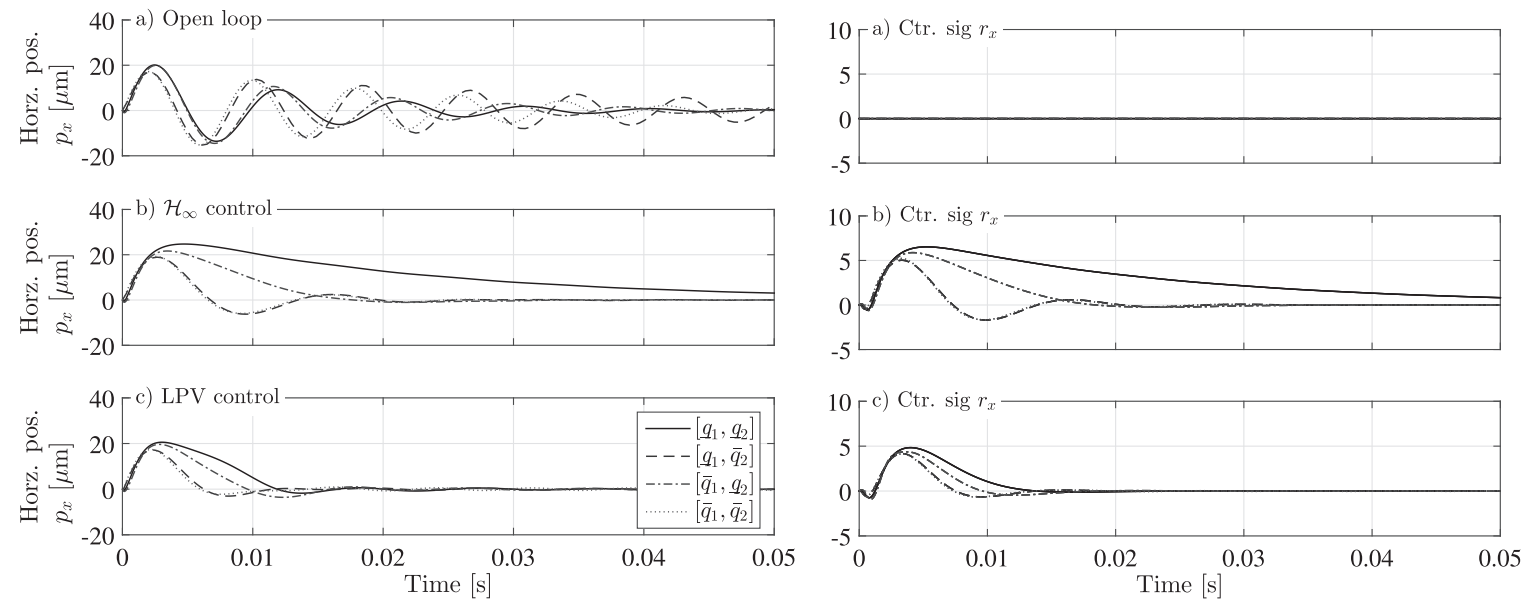

Fig. 10. Simulated horizontal impact responses for the scheduling parameter extrema in the three cases: (a) open loop, (b) $\mathcal{H}_{\infty}$ control, and (c) LPV control. Figures to the right show corresponding control signals.

\section{2. $\mathcal{H}_{\infty}$ controller design}

The LPV controller can directly address the parameter-dependency, though conservatism may be introduced by constraining the controller parameters to polynomial surfaces. A simpler control design methodology able to address the multiple objectives is the $\mathcal{H}_{\infty}$ control.

The design methodology offers several possibilities. The parameter-varying dynamics could be modelled as multiplicative uncertainty. Such approach will result in a conservative design since performance is required in the frequency range of the under-damped eigenvalues where the gain varies strongly. The controller could therefore not be able to sufficiently enhance the damping in the desired frequency range. An alternative and simpler approach is to design the controller for one operating condition to pursue a high damping increase for that nominal plant. This approach only guarantees stability and performance for the design condition. Therefore further investigation over the selected operating range must be carried out numerically.

The controller must be designed from a non-parameter-varying reference model that is chosen as the gas bearing model evaluated in the centre operating condition $\mathbf{q}_{0}=[3 \mathrm{krpm}, 0.5 \mathrm{MPa}]^{\mathrm{T}}$. The same mixed sensitivity set-up for the LPV controller is used with the weights evaluated at $\mathbf{q}_{0}$ to have $\mathbf{N}\left(s, \mathbf{q}_{0}\right)=\mathbf{N}(s)$

$$
\mathbf{K}(s)=\underset{\gamma}{\operatorname{argmin}}\|\mathbf{N}(s)\|_{\infty}, \quad \mathbf{N}(s)=\left[\begin{array}{c}
\mathbf{W}_{p}(s) \mathbf{S}(s) \\
\mathbf{W}_{u}(s) \mathbf{K}(s) \mathbf{S}(s)
\end{array}\right],
$$

The controller is then optimised for this particular operating condition and a performance index $\gamma_{\mathcal{H}}=1.13$ is achieved.

The stability and performance of the $\mathcal{H}_{\infty}$ controller over the operating range is then validated by calculating the closed-loop system over $Q_{v}$, and it is found that the controller stabilises the gas bearing. The worst case performance index is found to be $\max \gamma=2.28$ at $\mathbf{q}=[1.86 \mathrm{krpm}, 0.3 \mathrm{MPa}]^{\mathrm{T}}$. Fig. 9 shows the performance in the validation grid $Q_{v}$ of the $\mathcal{H}_{\infty}$ in comparison to the LPV controller. The LPV controller performance varies little over the design interval. Based on the $\mathcal{H}_{\infty}$ performance values, the LPV controller in general outperforms the $\mathcal{H}_{\infty}$ controller except for a range around the design condition of the $\mathcal{H}_{\infty}$ controller. This is expected since the LPV controller gains are constrained to polynomial surfaces and similar results were found in $[16,17]$.

\section{Numerical validation of LPV and $\mathcal{H}_{\infty}$ controllers}

The performance of rotating machines is often assessed using impulse responses that effectively reveals under-damped modes by exciting the system over a wide frequency range. Such impulse responses have been generated for a fine grid of operating conditions. The impulse magnitude is selected to correspond to a strong impact. Due to spacial limitations, four responses are included in the paper as they are representative of the overall impulse responses. The shown results correspond to the gas bearing operating at the four scheduling parameter extrema $\left\{\left[\underline{q}_{1}, \underline{q}_{2}\right]^{\mathrm{T}},\left[\bar{q}_{1}, \underline{q}_{2}\right]^{\mathrm{T}},\left[\underline{q}_{1}, \bar{q}_{2}\right]^{\mathrm{T}},\left[\bar{q}_{1}, \bar{q}_{2}\right]^{\mathrm{T}}\right\}$.

The impulse responses are compared for three different cases: (a) open-loop operation, (b) $\mathcal{H}_{\infty}$ controlled, and (c) LPV controlled. Both output directions have been investigated over $Q_{v}$ showing that the controller performance of the LPV and the $\mathcal{H}_{\infty}$ controller are similar in the vertical direction, whereas the horizontal direction shows more variation. Therefore only the horizontal results are illustrated in Fig. 10. 


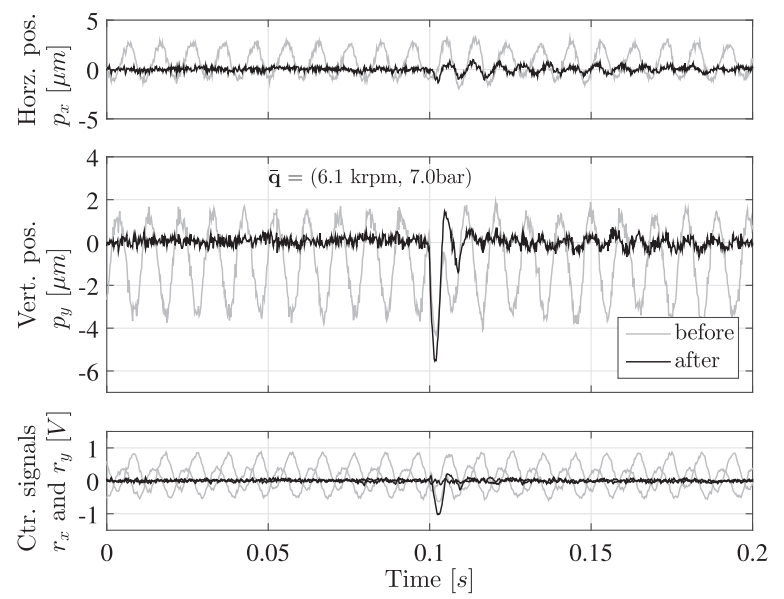

Fig. 11. Closed-loop impact response at $\mathbf{q}=[6 \mathrm{krpm}, 0.7 \mathrm{MPa}]^{\mathrm{T}}$. To only display the impact response run-out filtering is applied to remove the effect of the mass imbalance.

The impulse responses in open-loop show differences in the natural frequency and the damping factor emphasizing the strong dependency of dynamical behaviour on operating conditions. The $\mathcal{H}_{\infty}$ controller is able to enhance the damping in the investigated cases, however the LPV controller achieves a higher damping enhancement as the reduced settling time and peak magnitude demonstrate. This is clearly seen when comparing the closed-loop responses at the lowest rotational speed and injection pressure. The horizontal shaft displacement of the $\mathcal{H}_{\infty}$ regulated system peaks above $20 \mu \mathrm{m}$ and it has a slow decaying transient; on the contrary the LPV controller keeps $p_{x}$ below $20 \mu \mathrm{m}$ and it drives the displacement to zero in about 20 ms thanks to the gain scheduling across the system operating range. For the chosen impulse force, control signal saturation would occur in the $\mathcal{H}_{\infty}$ controlled case as $r_{x}$ exceeds the saturation limits $r_{x} \in[-5,5] \mathrm{V}$. This would further deteriorate the performance of the $\mathcal{H}_{\infty}$. The LPV control signal remains instead within the limits for all tested cases.

\section{Experimental validation of LPV and $\mathcal{H}_{\infty}$ controllers}

This section presents the findings of the extensive experimental campaign conducted in order to validate the capability of the two robust controller of enhancing the damping properties of the gas bearing system. Impact responses were carried out on the test-rig presented in Section 2 both in uncontrolled and controlled conditions and the collected responses are used to assess the performance of the $\mathcal{H}_{\infty}$ and LPV regulators. Last, experiments were performed during angular velocity transients to investigate the controllers' regulation capability during variations of one scheduling parameter.

To implement the designed control systems in the dSpace environment the controllers must be discretised. The $\mathcal{H}_{\infty}$ controller is converted to discrete time using a Tustin approximation. The LPV controller is discretised using the trapezoidal LPV discretisation method proposed in [23]. The calculation of the LPV controller matrix updates requires the inversion of two matrices of dimensions $12 \times 12$, which results in a high computational burden. Therefore it was decided to update the LPV controller gains at a lower frequency as proposed in [15]. The available hardware allows the controller matrices to be updated with a sampling rate of $10 \mathrm{~Hz}$ while the control signal is updated with the sampling rate $f_{s}=5 \mathrm{kHz}$. Since variations of the scheduling parameters occur on a time scale of seconds, the updating rate of the LPV controller matrix is more than sufficient to keep track of those changes.

\subsection{Impact responses}

The controllers' performance is experimentally investigated by means of impulse responses similar to those described in Section 4 since they are effective for exciting the system eigenfrequencies. The shaft was therefore excited at locations as close as possible to the gas bearing with an impact hammer that measured the applied force $f(t)$. Such impulse responses were collected over a fine grid of operating conditions chosen as the combination of angular velocities $q_{1} \in\{0,1.5,3,4.5,6\} \mathrm{krpm}$ and injection pressures $q_{2} \in\{0.3,0.43,0.57,0.7\} \mathrm{MPa}$. This included points different from the identification grid to ensure robust regulation in the interpolation regions too.

The impact response data collected for rotating conditions contains both the contribution from mass imbalance and from the shaft impact. The displayed data has therefore been filtered to remove the mass imbalance response. An example of data before and after filtering is shown in Fig. 11.

The experimental closed-loop results resemble the simulated ones and both controllers enhance the damping significantly. The experiments show that the LPV and the $\mathcal{H}_{\infty}$ controllers achieve similar performance in the vertical shaft 
a)
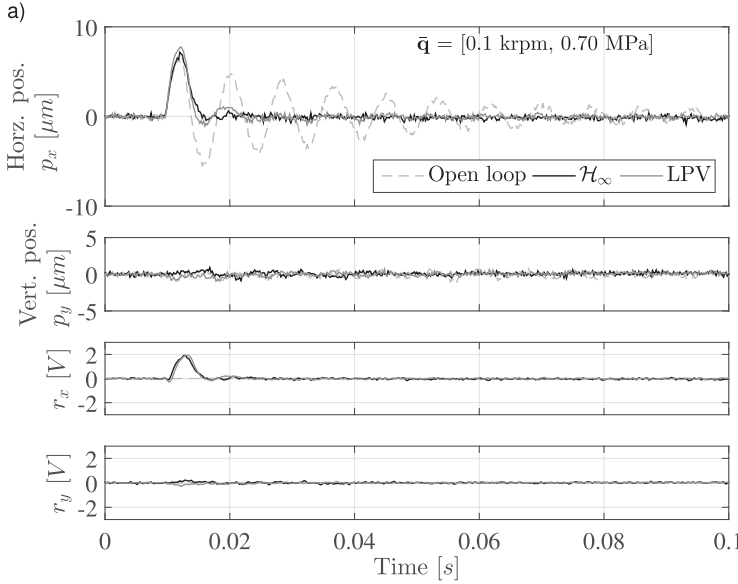

c)
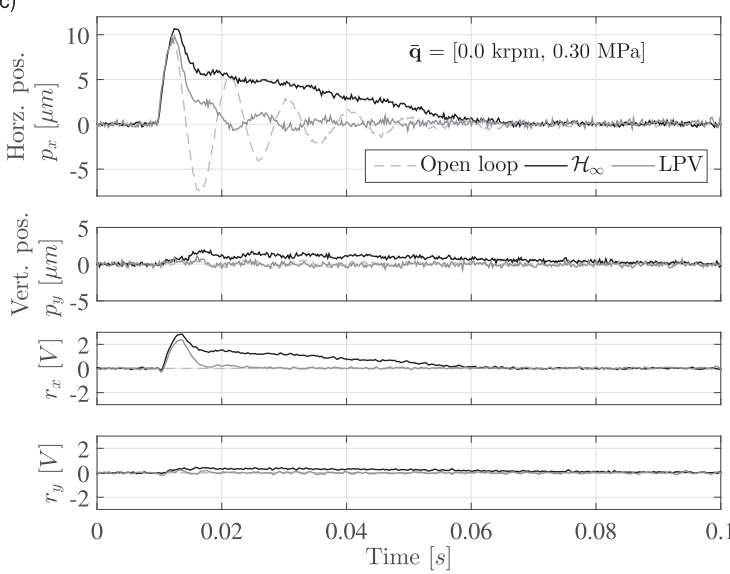

b)
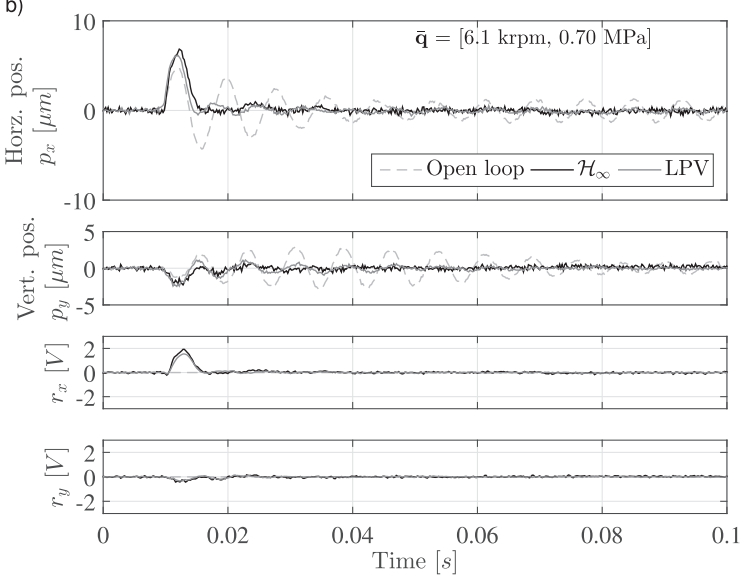

d)
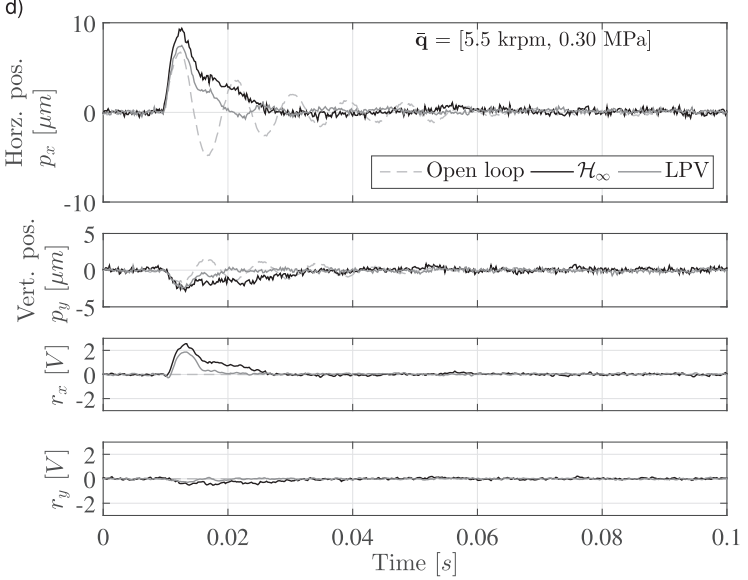

Fig. 12. Horizontal open- and closed-loop impact responses at the scheduling parameters extrema.

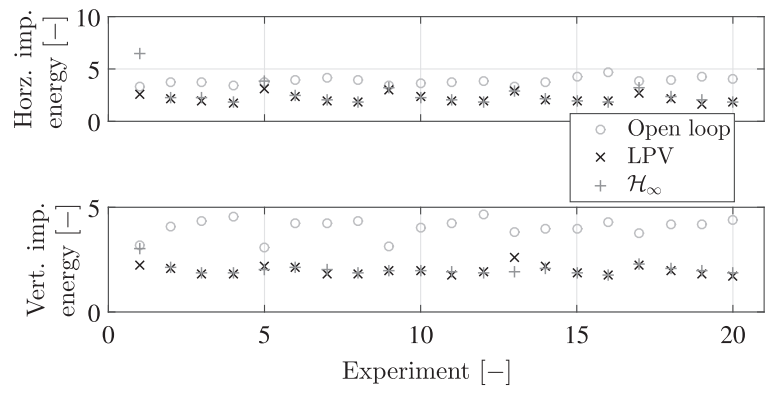

Fig. 13. Mean energy for the open- and closed-loop impact responses calculated with (25).

direction whereas differences are more evident in the horizontal direction. Due to the space restrictions all the $4 \times 5 \times 3$ impact responses cannot be shown in the paper, but Fig. 12 shows filtered horizontal impact responses for the open-loop, $\mathcal{H}_{\infty}$ controlled and the LPV controlled cases at the scheduling parameter extrema. The controllers enhance the damping in all cases. Four vertical impact responses at the operating conditions $q_{1} \in\{1.5,4.5\} \mathrm{krpm}, q_{2} \in\{0.433,0.567\} \mathrm{MPa}$ are shown in Appendix A to validate that the performance is also achieved in off-identification grid points.

It is desired to quantify the controllers performance both in terms of damping enhancement and noise rejection. A common approach is to estimate the damping factors using e.g. the logarithmic decrement. This approach is not suitable here since the controller increases the total system order by 12 and the impact responses cannot easily be decomposed. Therefore alternative approaches must be sought. When the damping is increased the impulse response decays faster hence the energy of the impulse response decreases. The magnitude of the response is proportional to the impact energy. The impulse responses are therefore quantified with the ratio of the disc displacement energy and the impact force energy denoted as 


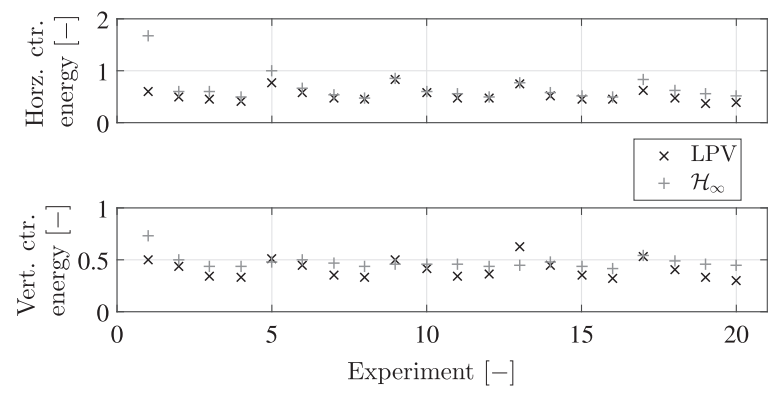

Fig. 14. The mean control efforts for closed-loop impact responses calculated with (27).

Table 3

Mean response energies of impact responses.

\begin{tabular}{llll}
\hline Mean energy $\backslash$ Experiment & Open-loop & LPV & $\mathcal{H}_{\infty}$ \\
\hline Horizontal impact energy & 14.94 & 5.08 & 7.63 \\
Vertical impact energy & 16.51 & 4.00 & 4.20 \\
Horizontal control energy & & 0.30 & 0.53 \\
Vertical control energy & & 0.18 & 0.23 \\
\hline
\end{tabular}
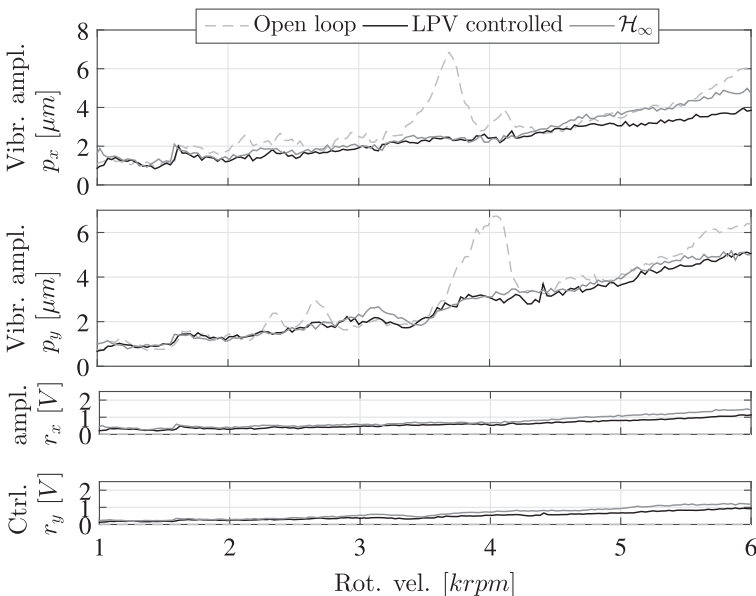

Fig. 15. Vibration amplitude in open loop, with LPV and $\mathcal{H}_{\infty}$ controllers during run-up and run-down in the interval $q_{1} \in[0,6] \mathrm{krpm}$ at $q_{2}=0.7 \mathrm{MPa}$.

$$
V_{p}=\frac{\|\mathbf{p}(t)\|_{\mathcal{L}_{2}}}{\|f(t)\|_{\mathcal{L}_{2}}}
$$

where the $\mathcal{L}_{2}$-norm of a signal $\mathbf{a}(t)=\left[a_{1}(t), a_{2}(t), \ldots, a_{J}(t)\right]^{\mathrm{T}}$ sampled at instants $k T_{s}, k \in\{0,1, \ldots, K\}$ is

$$
\|\mathbf{a}(t)\|_{\mathcal{L}_{2}}=\sqrt{\frac{1}{T_{s}} \sum_{k=0}^{K} \sum_{j=1}^{J} a_{j}^{2}\left(k T_{s}\right)}
$$

The impact responses are defined as settled after $K=500$ samples. Similarly, the control effort is quantified as the energy $V_{p}$ defined as the ratio of the control signal displacement energy and the force energy:

$$
V_{r}=\frac{\|\mathbf{r}(t)\|_{\mathcal{L}_{2}}}{\|f(t)\|_{\mathcal{L}_{2}}}
$$

Multiple impact responses have been collected for each experimental grid point and the mean energy for the impact responses at the 20 experimental grid points is shown in Fig. 13. The LPV controller reduces the response energy in all cases whereas the $\mathcal{H}_{\infty}$ controller reduces the energy at all operating conditions except Experiment 1 at $\boldsymbol{q}=[0.0 \mathrm{krpm}, 0.3 \mathrm{MPa}]$. This is in good agreement 
with the long settling time observed in Fig. 12c. The average control efforts of the LPV and $\mathcal{H}_{\infty}$ controllers in Fig. 14 show that the LPV controller generally has the lowest control effort as expected from Fig. 10. In Experiment 1 the $\mathcal{H}_{\infty}$ controller has a high effort, which is expected from the slow transient behaviour shown in Fig. 12c.

The average impact and control energies over the entire experimental data set were computed and Table 3 summarises the results. The $\mathcal{H}_{\infty}$ controller in average reduces the impact energy for horizontal impacts of $49 \%$ compared to the open-loop case and the LPV controller in average reduces the impact energy of $66 \%$. Similarly for the vertical impacts, the $\mathcal{H}_{\infty}$ controller reduces the impact energy of $75 \%$, and the LPV controller of $76 \%$. The LPV controller reduces the control effort compared to the $\mathcal{H}_{\infty}$ controller of $43 \%$ for the horizontal impacts and $22 \%$ for the vertical ones. Differences in performance between the horizontal and vertical directions are due to the different eccentricity of the shaft along the two axis. In the vertical direction due to the gravity pull the shaft is not located at the central position, which determines higher aerodynamic forces that guarantee greater controllability of the system. Conversely in the horizontal direction the shaft position is almost located at the centre position, which entails a reduced controllability in that direction. This is in line with previous results discussed in [7].

These results show that the LPV controller clearly benefits from an updated knowledge of the system's dynamics across the operational envelope, which enhances its performance in comparison with the $\mathcal{H}_{\infty}$ regulator particularly in the horizontal direction.

The experimental results are interesting since the $\mathcal{H}_{\infty}$ controller was tuned for one operating condition to pursue a high attenuation for the nominal plant. Fig. 9 shows that this comes at the cost of performance in the "global sense" since its performance deteriorates significantly over the operating range. A controller designed to guarantee robust performance would even locally be outperformed by the LPV controller. The approach pursued was very simple and the resulting controller was effective over the operating range.

\subsection{Run-up and coast-down}

During start-up and shut-down the rotating machine is subject to faster variations in angular velocity due to acceleration and deceleration phases. The LPV and $\mathcal{H}_{\infty}$ controllers were not designed to guarantee robustness and performance for fast changes in the scheduling parameters; however simulations have shown that the robust regulators are effective in these scheduling parameter transients as well. Experiments have therefore been performed to evaluate the controllers capabilities during these transient modes. During the experiments the shaft is accelerated from $0 \mathrm{krpm}$ to $6 \mathrm{krpm}$ with a mean acceleration of $0.1 \mathrm{krpm} / \mathrm{s}$. The turbine supply is then turned off and the shaft decelerates at a mean rate of $0.04 \mathrm{krpm} / \mathrm{s}$. Fig. 15 shows the vibration amplitude during the run-up and following coast-down phase both for the open-loop and the controlled gas bearing using both controllers.

At low angular velocities the controllers amplify the vibrations within an acceptable level as expected from the design. As the angular velocity increases the controllers reduce the vibration amplitude compared to open-loop. At $6 \mathrm{krpm}$ the amplitude of the vibrations in the horizontal direction is reduced of $37.4 \%$ by the LPV controller and of $23.1 \%$ by the $\mathcal{H}_{\infty}$ regulator. Once again equal performance is achieved in the vertical direction with a vibration amplitude reduction of $93 \%$. As desired both controllers use limited control efforts to avoid wear.

The open-loop experiments show vibration peaks in the horizontal and the vertical direction around angular velocities $3.69 \mathrm{krpm}(=61.5 \mathrm{~Hz})$ and $4.05 \mathrm{krpm}(=67.5 \mathrm{~Hz})$ and also at $2.34 \mathrm{krpm}(=39.0 \mathrm{~Hz})$ and $2.64 \mathrm{krpm}(=44.0 \mathrm{~Hz})$. These frequencies correspond to one half and one third of the first critical speeds. Such vibrations commonly indicate that the rotor is not accurately mounted in the gas bearing, i.e. there is a misalignment. The misalignment is difficult to avoid in applications with low tolerances such as gas bearings. This causes excitation at integer multiples of the angular velocity $\left(q_{1}, 2 q_{1}, 3 q_{1}, \ldots\right)[24-26]$. Inspection of the frequency content supports the hypothesis of misalignment. The misalignment vibrations are significantly amplified around the under-damped eigenfrequencies, which the controllers effectively reduce with a limited control effort.

\section{Conclusions}

Gas bearings appear as an appealing technology in the operation of high-speed rotating machines, particularly for the low environmental footprint and low energy losses. However this comes at the expense of modest damping properties, which limit the operational range of the gas bearing and in turn of the rotating machine. This work has addressed this issue proposing the enhancement of the damping properties through active control of the injection pressure. Two robust controllers have been designed utilizing state-of-the-art methods within the linear parameter-varying control theory and the $\mathcal{H}_{\infty}$ control theory. The controllers differ for the complexity of the design methodology and for the computational burden involved in computing the regulation signal.

The performance of the designed robust controllers has been extensively tested through an experimental campaign conducted on a scale model of a gas bearing. The experimental results pointed out the following major elements:

- The LPV and the $\mathcal{H}_{\infty}$ controllers equally perform in stabilizing vertical shaft vibration, although the $\mathcal{H}_{\infty}$ regulator does it with $27 \%$ more control effort in average.

- The LPV controller clearly outperforms the $\mathcal{H}_{\infty}$ controller in stabilizing horizontal shaft vibrations. In particular the LPV regulator reduces the amplitude of the lateral vibrations of $17 \%$ more than the $\mathcal{H}_{\infty}$ controller in comparison to the open-loop responses.

- Both the LPV and the $\mathcal{H}_{\infty}$ controllers guarantee stability and performance of the gas bearing system during start-up and 
shut-down phases, when parameter variation occurs at higher rate than considered at the controllers design stage.

- Experimental results are in good agreement with the numerical analysis of the closed-loop system, which implies the suitability of the available LPV model [7] of the gas bearing system for model-based control system design.

The experimental campaign validates the designed robust controllers as viable solutions for the enhancement of the damping characteristics of the gas bearing, and shows the feasibility of applying this technology in industrial settings where demands for high throughput and high reliability need to be met.

\section{Acknowledgements}

Prof. Hendricks from DTU Electrical Engineering is gratefully acknowledged for his feedback on the manuscript. The Danish Ministry of Science, Innovation and Higher Education is gratefully acknowledged for the financial support to the FTP research project $12-127502$.

\section{Appendix A. Vertical impact responses}

This appendix contains the comparison of vertical impact responses of the LPV controlled and the open loop gas bearing for four non-identification operating conditions. During these experiments, the shaft was excited from the top of the shaft, and the first oscillation is therefore negative. The displayed responses have been filtered to remove the mass imbalance response. The responses are shown in Fig. A1. The controllers effectively enhance the damping.
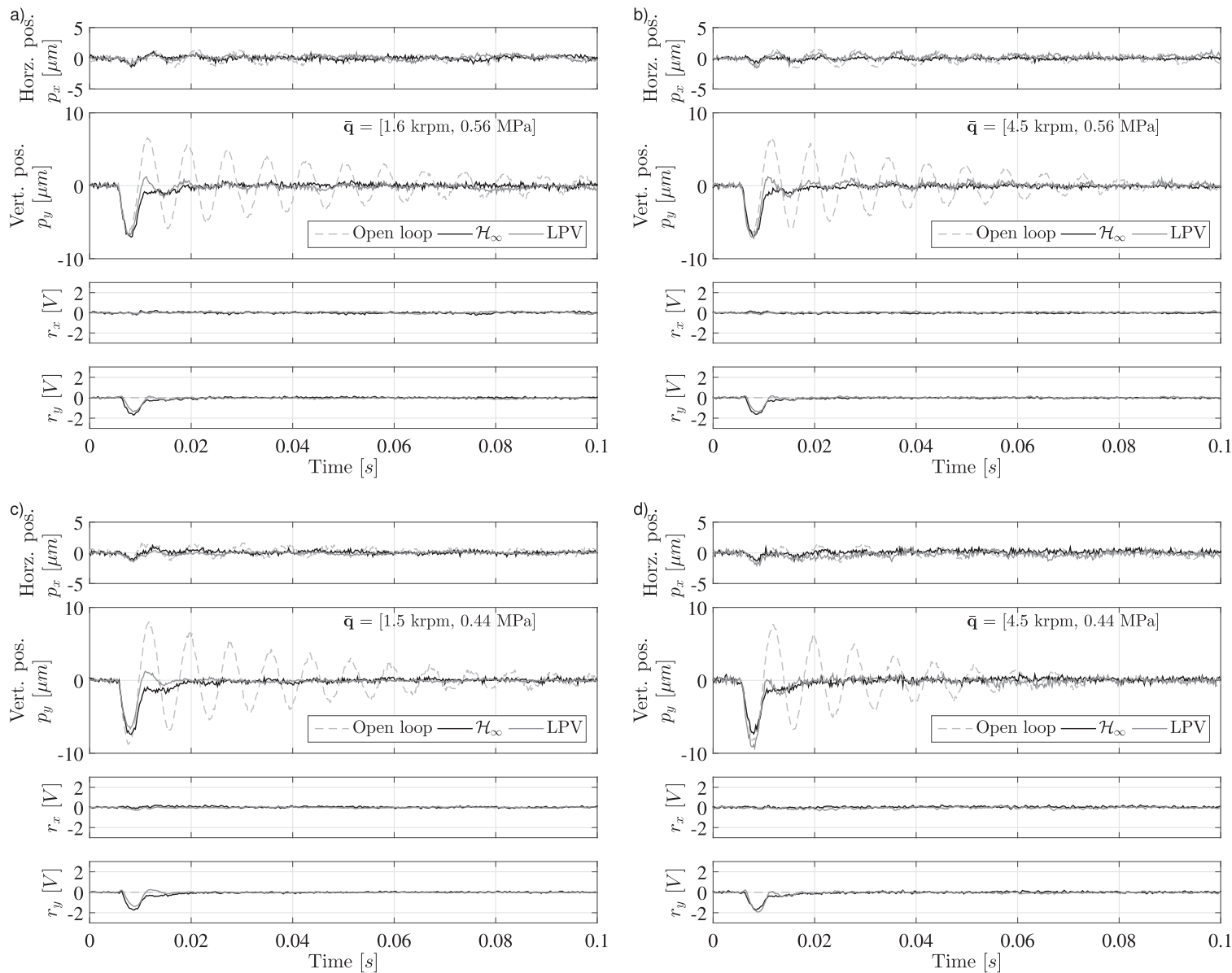

Fig. A1. Vertical open loop and closed-loop impact responses at the four operating conditions $\{7,9,12,14\}$ : (a) $\mathbf{q}=[1.5 \mathrm{krpm}, 0.57 \mathrm{MPa}]^{\mathrm{T}}$, (b) $\mathbf{q}=[4.5 \mathrm{krpm}, 0.57 \mathrm{MPa}]^{\mathrm{T}}$, (c) $\mathbf{q}=[1.5 \mathrm{krpm}, 0.43 \mathrm{MPa}]^{\mathrm{T}}$, and $(\mathrm{d}) \mathbf{q}=[4.5 \mathrm{krpm}, 0.43 \mathrm{MPa}]^{\mathrm{T}}$. 


\section{Appendix B. Grid points}

The scheduling parameters for which models were identified are listed in Table B1.

Table B1

$7 \times 5$ Grid for model identification $\left(q_{1} \times q_{2}\right)$.

\begin{tabular}{lllllll}
\hline$\left(q_{1}\right)[\mathrm{krpm}]$ & 0.00 & 1.00 & 2.00 & 3.00 & 4.00 & 5.00 \\
$\left(q_{2}\right)[\mathrm{MPa}]$ & 0.30 & 0.40 & 0.50 & 0.60 & 0.70 & \\
\hline
\end{tabular}

The scheduling parameters used for LPV control design are listed in Table B2.

Table B2

$8 \times 8$ Grid for control design $\left(q_{1} \times q_{2}\right)$.

\begin{tabular}{lllllllll}
\hline$\left(q_{1}\right)[\mathrm{krpm}]$ & 0.00 & 0.86 & 1.71 & 2.57 & 3.43 & 4.29 & 5.14 & 6.00 \\
$\left(q_{2}\right)[\mathrm{MPa}]$ & 0.30 & 0.36 & 0.41 & 0.47 & 0.53 & 0.59 & 0.64 & 0.70 \\
\hline
\end{tabular}

Those used for validation of the LPV control design are listed in Table B3.

\section{Table B3}

$16 \times 16$ Grid for control design $\left(q_{1} \times q_{2}\right)$.

\begin{tabular}{lllllllll}
\hline$q_{1}[\mathrm{krpm}]$ & 0.00 & 0.40 & 0.80 & 1.20 & 1.60 & 2.00 & 2.40 & 2.80 \\
$q_{2}[\mathrm{MPa}]$ & 3.20 & 3.60 & 4.00 & 4.40 & 4.80 & 5.20 & 5.60 & 6.00 \\
& 0.30 & 0.33 & 0.35 & 0.38 & 0.41 & 0.43 & 0.46 & 0.49 \\
& 0.51 & 0.54 & 0.57 & 0.59 & 0.62 & 0.65 & 0.67 & 0.70 \\
\hline
\end{tabular}

The operating conditions for which experimental impulse responses are collected are listed in Table B4.

Table B4

$5 \times 4$ Grid for experimental impulse response collection $\left(q_{1} \times q_{2}\right)$.

\begin{tabular}{|c|c|c|c|c|c|}
\hline$q_{1}[\mathrm{krpm}]$ & 0.00 & 1.50 & 3.00 & 4.50 & 6.00 \\
\hline$q_{2}[\mathrm{MPa}]$ & 0.30 & 0.43 & 0.57 & 0.70 & \\
\hline
\end{tabular}

\section{References}

[1] A.Z. Szeri, Low friction composite-film bearings, in: 2007 Proceedings of the ASME/STLE International Joint Tribology Conference, IJTC 2007, Part A, 2008, pp. 215-217.

[2] O. Horikawa, A. Shimokohbe, An active air bearing - (control of radial axis motion and stiffness), JSME Int. J. Ser. III-Vib. Control Eng. Eng. Ind. 33 (1) (1990) 55-60.

[3] H. Mizumoto, S. Arii, Y. Kami, K. Goto, T. Yamamoto, M. Kawamoto, Active inherent restrictor for air-bearing spindles, Precis. Eng. 19 (2-3) (1996) $141-147$.

[4] S. Morosi, I.F. Santos, Active lubrication applied to radial gas journal bearings. Part 1: Modeling. Tribol. Int. 44 (12) (2011) 1949-1958.

[5] J.S. Larsen, I.F. Santos, Compliant foil journal bearings-investigation of dynamic properties, in: Proceedings of the SIRM 2013, 10th International Conference on Vibrations in Rotating Machines, 2013.

[6] S. Morosi, I.F. Santos, Experimental investigations of active air bearings, Proceedings of Asme Turbo Expo 2012, vol. 7 (Parts A and B), 2012, pp. 901-910.

[7] L.R.S. Theisen, H.H. Niemann, I.F. Santos, R. Galeazzi, M. Blanke, Modelling and identification for control of gas bearings, Mech. Syst. Signal Process. 70-71 (2016) 1150-1170.

[8] O. Horikawa, H. Osada, A. Shimokohbe, An active air journal bearing, J. Jpn. Soc. Precis. Eng. 55 (11) (1989) 2063-2068.

[9] F. Pierart, I. Santos, Active lubrication applied to radial gas journal bearings. part 2: Modelling improvement and experimental validation, Tribol. Int. 96 (2016) 237-246. 
[10] L.R.S. Theisen, H.H. Niemann, I.F. Santos, R. Galeazzi, Experimental investigations of decentralised control design for the stabilisation of rotor-gas bearings, in: Proceedings of the XVII International Symposium on Dynamic Problems of Mechanics, 2015.

[11] F. Wu, X.H. Yang, a. Packard, G. Becker, Induced L_2-norm control for LPV systems with bounded parameter variation rates, Int. J. Robust Nonlinear Control 6 (1996) 983-998.

[12] P. Apkarian, R. Adams, Advanced gain-scheduling techniques for uncertain systems, IEEE Trans. Control Syst. Technol. 6 (1) (1998) 21-32.

[13] C. Hoffmann, H. Werner, A survey of linear parameter-varying control applications validated by experiments or high-fidelity simulations, IEEE Trans. Control Syst. Technol. 23 (2) (2015) 416-433.

[14] A.A.G. Siqueira, R. Nicoletti, N. Norrick, K.L. Cavalca, H. Fiori de Castro, J. Bauer, F. Dohnal, Linear parameter varying control design for rotating systems supported by journal bearings, J. Sound Vib. 331 (10) (2012) 2220-2232.

[15] J. Witte, H. Balini, C. Scherer, Robust and LPV control of an AMB system, in: American Control Conference, 2010, pp. 2194-2199.

[16] H.M.N.K. Balini, J. Witte, C.W. Scherer, Synthesis and implementation of gain-scheduling and LPV controllers for an AMB system, Automatica 48 (3) (2012) 521-527.

[17] S. Mason, P. Tsiotras, P. Allaire, Linear parameter varying controllers for flexible rotors supported on magnetic bearings, in: Proceedings of 6th International Symposium on Magnetic Bearings, 1998, pp. 341-351.

[18] S. Morosi, From hybrid to actively-controlled gas lubricated bearings - theory and experiment (Ph.D. thesis), Technical University of Denmark, 2011.

[19] I.F. Santos, Design and evaluation of two types of active tilting pad journal bearings, in: The Active Control of Vibration, 1994 , pp. 79-87.

[20] S. Skogestad, I. Postlethwaite, Multivariable Feedback Control: Analysis and Design, John Wiley \& Sons, New York, NY, USA, 2005.

[21] J.S. Freudenberg, D.P. Looze, Right half plane poles and zeros and design tradeoffs in feedback systems, IEEE Trans. Autom. Control 30 (6) (1985) $555-565$.

[22] J. Lofberg, YALMIP: a toolbox for modeling and optimization in Matlab, in: 2004 IEEE International Symposium on Computer Aided Control Systems Design, 2004, pp. 284-289.

[23] P. Apkarian, On the discretization of LMI-synthesized linear parameter-varying controllers, Automatica 33 (4) (1997) $655-661$.

[24] S.A. Howard, Misalignment in gas foil journal bearings: an experimental study, J. Eng. Gas Turbines Power 131 (2) (2009) 022501.

[25] A.S. Sekhar, B.S. Prabhu, Effects of coupling misalignment on vibrations of rotating machinery, J. Sound Vib. 185 (4) (1995) 655-671.

[26] D.L. Dewell, L.D. Mitchell, Detection of a misaligned disk coupling using spectrum analysis, J. Vib. Acoust. Stress Reliab. Des. 106 (1) (1984) 9. 